\title{
A Radiographic Analysis of Anatomical Variation at The Mandibular Sites of Intraoral Bone Harvesting
}

\section{CBCT risk assessment in mandibular grafting}

Key Words:

Donor site, CBCT, Mandible, Implant bone, Grafting

\author{
Authors and Institutions \\ Corresponding Author \\ Dr Robert Adams \\ School of Dentistry, Cardiff University CF14 4XY, UK \\ AdamsR6@ cardiff.ac.uk \\ Date of submission: 8/6/2017
}

Dr Robert Adams, School of Dentistry, Cardiff University CF14 4XY, UK Dr Luvay Hamid, School of Dentistry, Cardiff University CF14 4XY, UK Dr Adrian Binney, School of Dentistry, Cardiff University CF14 4XY, UK Dr Nicholas Claydon, School of Dentistry, Cardiff University CF14 4XY, UK Dr Damian Farnell, School of Dentistry, Cardiff University CF14 4XY, UK Dr Bridie Griffiths, School of Dentistry, Cardiff University CF14 4XY, UK Professor David Thomas, School of Dentistry, Cardiff University CF14 4XY, UK 


\section{Abstract}

Objectives: Intraoral bone grafting is an important component of implant treatment, but also a source of intra and postoperative complications. This study sought to determine the relationship of bone surface to underlying nerves and vasculature, at the two most commonly employed intraoral sites for bone harvesting.

Material and Methods: Data was collected by direct measurement of 103 potential graft sites (50 retromolar sites: 53 symphyseal sites) of patients undergoing CT scanning for implant surgery assessment.

Results: Symphyseal analysis included 26 female and 27 male patients. Data recorded included: maximal width of the mandible between lower central incisor root tips (mean $13.7 \mathrm{~mm}$; range $11.3-16.4 \mathrm{~mm}$ ), maximal width of the mandible below the canine tip (mean $11.5 \mathrm{~mm}$; range $7.7-14.9 \mathrm{~mm}$ ), tip of canine to mandibular incisive nerve (mean 4.3mm; range $1.2-8.9 \mathrm{~mm}$ ), canine root tip to outer labial cortex (mean $4.0 \mathrm{~mm}$; range $1.9-7.3 \mathrm{~mm}$ ) and midline distance from the incisive nerve to outer labial cortex (mean 3.0mm; range $1.0-6.9 \mathrm{~mm}$ ). Retromolar analysis comprised of 33 female and 17 male patients. Data recorded included distance from: inferior dental canal to the outer buccal cortex (mean $3.2 \mathrm{~mm}$; range $1.0-7.9 \mathrm{~mm}$ ), the most buccal point of the lower second molar roots to the outer buccal cortex (mean 3.3mm; range $1.0-6.5 \mathrm{~mm}$ ) and thickness of the mandibular crest at the lower second molar (mean 13.8 ; range $10.2-18.2 \mathrm{~mm})$.

Conclusions: The proximity of underlying vital structures may be easily overlooked in routine examination of implant patients and may be related to patient characteristics.

\section{Clinical Relevance}

\section{Scientific rationale for study}

Intraoral bone grafting from the mandibular retro molar and symphyseal sites are common surgical procedures. Each has an associated level of complication and morbidity, which is related to the proximity of neighbouring anatomical structures. Currently, there is little documentation on the variation of these important anatomical structures. 


\section{Principal Findlings}

The main findings confirm the wide variation in the position and size of the anatomical structures associated with intraoral bone graft donor sites.

\section{Practical implications}

The study has highlighted the importance of good radiological assessment of these structures, prior to undertaking intraoral bone graft procedures, in order to provide informed consent.

\section{Introduction}

Bone augmentation is recognised as an important component of contemporary implant treatment. Bone augmentation facilitates optimisation of implant placement and optimisation of the aesthetic and functional outcomes of treated cases. There has been increased interest in the field of oral implantology in bone (and soft tissue) augmentation, and this is reflected in the rise in the number of publications in this subject area in the past two decades. ${ }^{1}$ Current techniques employed in soft tissue regeneration are well defined; having been established in periodontology and in the management of the peri implant soft tissues. ${ }^{2}$ In contrast, bone grafting techniques have continuously evolved in the last decade. Bone augmentation or grafting however, includes both a wide range of clinical indications e.g. vertical and horizontal augmentation, sinus lifting ${ }^{3}$ and (more recently) alveolar ridge preservation, ${ }^{4,5}$ with a wide range of techniques and technologies being employed. These techniques are diverse, clinically dependent, operator preferenced, and material dependent. The range of potential graft materials for use in implant surgery is extensive; ranging from patient derived allogenic bone to xenogeneic hydroxyapatite. The latter materials predominating in both the published literature and in the clinical market. ${ }^{1}$ The use of biomaterials to augment hard tissue has obvious advantages such as a decreased complexity of treatment and surgical time, together with osteoconductive properties. However, hard tissue augmentation using biomaterials alone has limitations in the size of the defect that may be reliably treated, ${ }^{6}$ the possibility that graft material may be incompletely resorbed ${ }^{7}$ or induce a foreign body reaction to the biomaterial. ${ }^{8}$

The use of autogenous bone in hard tissue augmentation in implant surgery remains attractive because it represents an osteoconductive and osteoinductive matrix. 
Furthermore, autogenous bone grafts contain growth factors that bind to the extracellular matrix of the bone, which in turn resist the proteolytic degradation of the inflammatory phase of healing post surgery. Autogenous bone grafting may be used to treat intraoral defects and it was first described in the field of oral and maxillofacial surgery almost 100 years ago. ${ }^{9}$ This procedure has remained popular since this time. Initially, the majority of autogenous bone grafts were extra oral, although it has become a useful tool in implant dentistry. The sites most typically utilised in implant dentistry as sources of bone tissues have included the iliac crest, ${ }^{10}$ tibia $^{11}$ and calvarium. ${ }^{12}$ As in soft tissue grafting, the techniques for intraoral bone grafting have evolved over time. Extraoral sites (e.g. the iliac crest) are now more typically reserved for cases of alveolar reconstruction in cases of extreme resorption, surgical resection, or following extensive direct trauma. Moreover, the relative merits of extraoral grafting (and its limitations) have been described extensively in the literature, including: cost; the need for general anaesthesia; and, donor site morbidity. ${ }^{13}$ The case of donor site morbidity is an important consideration, ${ }^{14}$ and it may include immobility in the case of iliac crest and tibial grafts. In addition, increased resorption in grafts from extraoral sites has been described previously, and this effect has been attributed to the embryological origins of the grafted bone. ${ }^{15,16}$

Intraoral bone harvesting for grafting in implantology has been extensively described from a variety of sites incorporated in a number of surgical techniques. The sites described have included the anterior nasal spine ${ }^{17}$ and tuberosity ${ }^{18}$ of the maxilla and the chin, body and ramus of the mandible. ${ }^{19}$ Due to improvements in techniques and appreciation of material and physiological limitations of GBR, intraoral block grafts are an essential treatment option in alveolar grafting. Significant complications associated with grafting have, however, been described. These complications include incomplete healing or healing with fibrous encapsulation of the graft. Donor site morbidity in intraoral block grafting is an important complication that can affect up to $40 \%$ of patients. ${ }^{20}$ The use of block grafts have a considerable advantage due to the volume of bone that can be harvested under local anaesthesia in an outpatient dental setting, reduced material costs and the avoidance of general anaesthesia. ${ }^{13}$ As well as the problems of failure of healing (and of the primary stability of the implant), significant donor site complications are well described and include both intraoperative (bleeding) and postoperative complications e.g. pain, swelling and numbness of the adjacent teeth and soft tissues due to neuropraxia or neurotemesis associated with the 
surgery. ${ }^{20}$

Although a number of the complications are operator dependent (e.g., flap design, retraction and duration of surgery), anatomical variation at the donor site is of paramount consideration in the consideration of risk of long term postoperative problems. A good understanding of the local donor site anatomy is pivotal in determining outcomes and planning surgery. The likelihood of nerve damage with respect to the local mandibular anatomy (and the absolute volume of bone that can be harvested) is anatomically constrained by the site of the neurovascular bundle and its relationship to the external bony surface. An accurate assessment of these relationships is essential in planning grafting and is increasingly important in a litigious society. Although we know something of variations in the course of the inferior dental nerve and its terminal branches, ${ }^{21,22,23,24}$ the relationship between the bone surface and the underlying neurovascular bundle at the sites of mandibular graft sites has not been characterised to date. One study has investigated the relationship of the bone to the underlying neurovascular tissues; these factors might not only affect success but also that they might be associated with donor site morbidity. ${ }^{20}$

In this study, we characterised the anatomy at two of the most commonly employed, intraoral block graft sites (namely, the chin and the ramus). We determined the anatomical variation in the bone thickness and neurovascular depth beneath the buccal surface. We determined factors within the patient demographic characteristics (namely, gender and age) that relate to the available volume of bone, and so to possible complications in graft surgery.

\section{Material and Methods}

Patients were attending for implant surgery at a Centre in Cardiff and were selected if they required a cone beam $\mathrm{CT}(\mathrm{CBCT})$ scan as part of their preoperative planning for dental implant surgery. The CBCT scan data was analysed by a single investigator (LH). All scans were anonymised prior to the investigation. All CBCT scans were analysed obtained using a Sirona GALILEOS CBCT scanner. Figure 1 shows a typical image obtained using the GALILEOS scanner. Patients were excluded from analysis in the study if their scans in the retromolar region demonstrated the presence of lower third molar teeth, supraeruption or periodontal pathology affecting the lower second molar teeth (e.g., cysts, or periodontal disease affecting the adjacent second 
molar teeth). Patients were also excluded if their scans demonstrated the presence of mandibular tori, or local pathology (vide supra) in the mandibular symphyseal region.

\section{Radiographic data analysis}

\section{Mandilbular Symphyseal anallyses}

The measurements taken at the mandibular symphyseal region are shown in Figs. (2a) and (2b). Measurements recorded at the canine region (Fig. 2a) included the maximum width of the mandible (GF) and the distance from the canine tip to the outer aspect of the buccal cortex (AC). In relation to the terminal incisive branch of the inferior dental nerve, measurements were recorded from the canine tip to the most superior aspect of the bone canal $(\mathrm{AB})$ and the buccal aspect of the canal to the outer aspect of the buccal cortex (HD). Measurements included the maximum width of the mandible below the apices of the lower first incisor (LM; Fig. 2b),

\section{Mandibular Retromolar Analyses}

The measurements recorded at the retromolar site are shown in Fig. 3. Measurements included the width of the mandible at the crest at the position of the second molar (GI) and the distance from the most buccal aspect of the roots of the second molar to the outer cortex of the buccal plate (DE). In an attempt to standardise the measurement of the position of the inferior dental nerve, a point was chosen at $10 \mathrm{~mm}$ $\pm 0.5 \mathrm{~mm}$ distal to the distal aspect of the lower second molar (position B). This point was chosen to represent a realistic position that would be involved in the harvesting of a retromandibular block graft. Other measurements taken included the vertical position of the inferior dental canal in relation to position B (BC), width of alveolus at this position of the inferior dental canal (EF) and the distance from the inferior dental canal to the outer aspect of the buccal plate (DE)

\section{Statistical Anallyses}

Statistical analyses were performed using SPSS V20 and MS EXCEL. Descriptive statistics were obtained for age and all measurements of distance at both sites. Gender differences in means of all distance measurements were tested using an independent samples $t$ test for both the symphyseal and retromolar analyses. 


\section{Results}

\section{Mandibular Symphysis}

53 CBCT scans were studied in the mandibular symphyseal region. Of these scans 26 (49\%) were taken for female patients and 27 (51\%) for male patients. The mean age of the patients was 52.2 years (range 20 - 74 years). Results for measurements made in the mandibular symphysis region are shown in Table 1 . The mean maximum thickness of the mandibular symphysis at the midline was found to be $13.7 \mathrm{~mm} \pm$ $1.44 \mathrm{~mm}$ (range $11.3-16.4 \mathrm{~mm}$ ). The average thickness of the mandible at the apices of the canine tip was $11.5 \mathrm{~mm} \pm 1.85 \mathrm{~mm}$ (range $7.7 \mathrm{~mm}-14.9 \mathrm{~mm}$ ). The average distance of the canine tip from the outer aspect of the buccal plate at the mandibular symphysis was $4.0 \mathrm{~mm} \pm 1.11 \mathrm{~mm}$ (range $1.9 \mathrm{~mm}-7.3 \mathrm{~mm}$ ). The average distance from the outer aspect of the buccal plate to the buccal aspect of the incisive canal was $3.0 \mathrm{~mm} \pm 1.27 \mathrm{~mm}($ range $1.0 \mathrm{~mm}-6.9 \mathrm{~mm})$.

\section{Retromolar Region}

50 CBCT scans were studied of the retromolar sites. Of these 33 were female patients $(66 \%)$ and 17 male $(34 \%)$. The mean age of the patients was 54.1 years (range $25-$ 75 years). Results for measurements made in the retromolar region are shown in Table 2. The average width of the mandible at the crest was $13.8 \mathrm{~mm} \pm 1.98 \mathrm{~mm}$ (range between $10.2 \mathrm{~mm}$ and $18.2 \mathrm{~mm}$ ). This study also measured the width of the mandible at the position of the inferior dental canal. The mean thickness of the mandible at the position of the IDC was $7.6 \mathrm{~mm} \pm 1.47 \mathrm{~mm}$ (range $4.7-11.3 \mathrm{~mm}$ ). The difference in mean thickness of the between the crest and the level of the IDC was $6.2 \mathrm{~mm}$. The average depth of the IDC was $10.1 \mathrm{~mm} \pm 2.48 \mathrm{~mm}$ (Range $4.0 \mathrm{~mm}-$ $15.4 \mathrm{~mm})$. The distance from the outer aspect of the buccal plate to the IDC was on average $3.2 \mathrm{~mm} \pm 1.23 \mathrm{~mm}$ (range $1.0 \mathrm{~mm}-7.9 \mathrm{~mm}$ ). The thickness of bone buccal to the root of the second molar was on average found to be $3.3 \mathrm{~mm} \pm 1.27 \mathrm{~mm}$ (range $1.0 \mathrm{~mm}-6.5 \mathrm{~mm})$.

\section{Discussion}

There are a number of recognised techniques available for bone reconstruction prior to or concurrent with implant placement. Available bone graft materials include allogenic, xenogenic, synthetic and autogenous bone. All of these materials have 
osteoconductive properties. However, autogenous bone is often considered the "gold standard" because it has both osteogenic and osteoinductive properties. Misch first published the use of intraoral retromolar and symphyseal grafts in dental implantology. ${ }^{25}$ The use of mandibular symphysis and retromolar sites have since become established as the main intraoral donor sites for bone reconstruction in implantology. ${ }^{19}$

The results of this study demonstrate the wide variation in anatomy at the sites of common intraoral bone grafting sites. This effect will clearly affect the volume of bone that can be grafted from individual patients, and these findings are in accordance with other studies. A related study ${ }^{26}$ estimated an average symphyseal graft volume of $2.3 \mathrm{~cm}^{3}$ (range $1.7-2.8 \mathrm{~cm}^{3}$ ) and Montazem et al. ${ }^{27}$ estimated an average graft volume of $4.84 \mathrm{~cm}^{3}$ (range $3.25-6.50 \mathrm{~cm}^{3}$ ). Verdugo et al..$^{28}$ also estimated the retromandibular graft volume as $2.5 \mathrm{~cm}^{3}$ (range $1.8-3.0 \mathrm{~cm}^{3}$ ). Nkenke et al, ${ }^{29}$ measured the width of the mandible at the retromolar region during graft harvest procedures using Zielinsky calipers. They found that the average width of the mandible was $14.2 \mathrm{~mm}$ (range $10-17 \mathrm{~mm}$ ). ${ }^{29}$ Our study demonstrated similar results. These results demonstrate the degree of lingual undercut. These results are in keeping with a previous study, ${ }^{30}$ which found that the thickness of the basal bone was $5 \mathrm{~mm}$ less on average than the thickness of the crest in posterior regions of the mandible. A number of reported complications can occur following the harvesting of intraoral bone grafts ${ }^{19,20,29}$. These complications include inferior alveolar nerve injury and damage to adjacent roots. A very important consideration in the planning of bone graft harvesting is the position of vital structures.

The important considerations at the retromolar site are the position of the inferior alveolar nerve and the position of the second molar teeth. We found in our study that the average depth of the IDC was $10.1 \mathrm{~mm} \pm 2.48 \mathrm{~mm}$ (range $4.0 \mathrm{~mm}-$ $15.4 \mathrm{~mm})$. This result agrees with a previous study by Nkenke et al. ${ }^{29}$ that measured the average depth of the IDC as $11.0 \mathrm{~mm} \pm 2.2 \mathrm{~mm}$. The distance from the outer aspect of the buccal plate to the IDC was on average $3.2 \mathrm{~mm} \pm 1.23 \mathrm{~mm}$ (range $1.0 \mathrm{~mm}-$ $7.9 \mathrm{~mm})$. The thickness of bone buccal to the root of the second molar was on average found to be $3.3 \mathrm{~mm} \pm 1.27 \mathrm{~mm}$ (range $1.0 \mathrm{~mm}-6.5 \mathrm{~mm}$ ). The important anatomical considerations at the mandibular symphysis are the position of the apices of the lower anterior teeth and the incisive branch of the inferior alveolar nerve. Here we found that the average distance of the canine tip from the outer aspect of the buccal plate 
was $4.0 \mathrm{~mm} \pm 1.11 \mathrm{~mm}$ (range $1.9 \mathrm{~mm}-7.3 \mathrm{~mm}$ ). The average distance from the outer aspect of the buccal plate to the buccal aspect of the incisive canal was $3.0 \mathrm{~mm} \pm$ $1.27 \mathrm{~mm}$ (range $1.0 \mathrm{~mm}-6.9 \mathrm{~mm}$ ).

These findings show that there will be a large difference in available graft volume depending on the anatomy of the proposed donor site when undertaking intraoral grafting procedures. They demonstrate also that some patients carry a much greater risk of complications than others. The minimum distance of the root of the second molar from the buccal plate at the retromolar site was $1.0 \mathrm{~mm}$. The minimum distance of the IDC from the outer buccal plate was also $1.0 \mathrm{~mm}$. The minimum distance of the canine tip from the buccal plate at the symphyseal region was found to be $1.9 \mathrm{~mm}$, and the incisive branch of the IAN at the symphyseal region was $1.0 \mathrm{~mm}$. In these instances, it would be impossible to harvest a graft of even the minimal volumes, which have previously described. ${ }^{26,27,28}$ In addition to the intra and postoperative complications, the lack of available bone has significant impact on increasing volume at the recipient site and subsequent implant placement.

These results demonstrate the risks involved to adjacent vital structures in the harvesting of bone grafts, which may be apparent in some patients. It also clearly demonstrates the need for good clinical examination and appropriate imaging of the mandibular donor sites when planning intraoral bone graft procedures and the potential consideration of alternative strategies where the anatomy will not allow sufficient volume to be harvested. 


\section{Disclosure statements}

Dr. Adams has nothing to disclose.

Dr. Luvay has nothing to disclose.

Dr. Binney has nothing to disclose.

Dr. Claydon has nothing to disclose.

Dr. Farnell has nothing to disclose.

Dr. Griffiths has nothing to disclose.

Prof. Thomas has nothing to disclose. 


\section{References}

1. Sonigra N, A systematic review of the literature on the use of BioOss in Dental Implantology. MSc Thesis, University of Wales, Submitted September 2015

2. Thoma DS, Buranawat B, Hammerle CH, Held U, Jung RE. Efficacy of soft tissue augmentation around dental implants and in partially edentulous areas: a systematic review. J Clin Periodontol 2014 (Apr);41 Suppl 15:S77-9

3. Aghaloo TL \& Moy PK. Which Hard Tissue Augmentation Techniques Are the Most Successful in Furnishing Bony Support for Implant Placement? Int J Oral Maxillofac Implants 2007;22(SUPPL):49-70

4. Artzi Z, Nemcovsky CE. The application of de-proteinized bovine bone mineral for ridge preservation prior to implantation. Clinical and histological observations in a case report. J Periodontol 1998; 69(9): 1062-7

5. Sclar AG. Preserving alveolar ridge anatomy following tooth removal in conjunction with immediate implant placement. The Bio-Col technique. Atlas Oral Maxillofac Surg Clin North Am 1999; 7(2): 39-59

6. LE BT \& Borzabadi-Farahani A. Simultaneous implant placement and bone grafting with particulate mineralised allograft in site with baccal wall defects, a three-year follow-up and review of the literature. J CranioMaxillofac Surgery 2014; 42: 552-9

7. Becker W, Urist M, Becker BE, Jackson W, Parry DA, Bartold M, Vincenzzi G, De Georges D, Niederwanger M. Clinical and histologic observations of sites implanted with intraoral autologous bone grafts or allografts. 15 human case reports. J Periodontol. 1996 Oct; 67(10): 1025-33

8. Bannister SR \& Powell CA. Foreign Body Reaction to Anorganic Bovine Bone and Autogenous Bone With Platelet-Rich Plasma in Guided Bone Regeneration. Periodontol 2008;79: 1116-1120

9. Chubb G. Demonstration of Cases and Radiographs illustrating the Technique employed and Results obtained in the Repair of Fractured Mandible by means of the Free Autogenous Bone-graft. Proc R Soc Med. 1921; 14(Surg Sect): 81-3 
10. Branemark P-I, Lindstrom J, Hallen $\mathrm{O}$ et al. Reconstruction of the defective mandible. Scand J Plast Reconstr Surg 1975. 9: 116-128.

11. Catone CA, Reimer BL, McNeir D, Ray R. Tibia autogenous cancellous bone as an alternative donor site in maxillofacial surgery: a preliminary report. J Oral Maxillofac Surg 1992;50:1258-1263

12. Gary JJ, Donovan M, Garner FT, Faulk JE. Rehabilitation with calvarial bone grafts and osseointegrated implants after partial maxillary resection. A clinical report. J Prosthet Dent 1992; 62743-746

13. Jensen J \& Sindet-Pedersen. Autogenous mandibular bone grafts and osseointegrated implants for reconstruction of the severely atrophied maxilla: a preliminary report. $\mathbf{J}$ Oral Maxillofac Implants 1991; 49(12): 1277-87

14. Reissmann DR, Dietze B, Vogeler M, Schmelzeisen R, Heydecke G. Impact of donor site for bone graft harvesting for dental implants on health-related and oral healthrelated quality of life. Clin Oral Implants Res. 2013 Jun;24(6):698-705

15. Smith JD and Abramson M. Membranous v endochondral bone autografts. Arch Otolaryngol 1974; 99: 203-205

16. Zins JE, Whitaker LA. Membranous vs endochondral bone autographs. Implications for craniofacial reconstruction. Plast Reconstr Surg 1983; 72: 778-785

17. Cho Y-S, Hwang K-G, Park C-J. Postoperative effects of anterior nasal spine bone harvesting on overall nasal shape. Clin. Oral Impl. Res. 24, 2013, 618-622

18. Moenning JE, Graham LL. Elimination of mandibular labial undercut with autogenous bone graft from a maxillary tuberosity. J Prosthet Dent. 1986 Aug; 56(2): $211-4$

19. Misch CM. Comparison of intraoral donor sites for onlay grafting prior to implant placement. Int J Oral Maxillofac Implnts 1997; 12(6): 767-76

20. Clavero J \& Stefan Lundgren, ramus or Chin Grafts for Maxillary Sinus Inlay and Local Onlay Augmentation: Comparison of Donor Site Morbidity and Complications. Clinical Implant Dentistry and Related Research, 2003; 5 (3): 154-160. 
21. Kim ST, Hu KS, Song WC, Kang MK, Park HD, Kim HJ. Location of the mandibular canal and the topography of its neurovascular structures. J Craniofacial Surgery 2009 May; 20(3): 936-9

22. Nortjé CJ, Farman AG, Grotepass FW. Variations in the normal anatomy of the inferior dental (mandibular) canal: a retrospective study of panoramic radiographs from 3612 routine dental patients. British Journal of Oral Surgery, 1977; 15(1):55-63

23. Rosa MB, Sotto-Maior BS, Machado Vde C, Francischone CE. Retrospective study of the anterior loop of the inferior alveolar nerve and the incisive canal using cone beam computed tomography. Int J Oral Maxillofac Implants 2013; 28(2): 388-92

24. Makris N, Stamatakis H, Syriopoulos K, Tsiklakis K, van der Stelt PF. Evaluation of the visibility and the course of the mandibular incisive canal and the lingual foramen using cone-beam computed tomography. Clin Oral Implants Res 2010; 21(7): 766-71

25. Misch CM, Misch CE, Resnik R, Ismail YH. Reconstruction of maxillary alveolar defects with mandibular symphysis grafts for dental implants: a preliminary procedural report. Int J Oral Maxillofac Implants 1992; 7:360-6

26. Verdugo F, Simonian K, Smith McDonald R, Nowzari H. Quantitation of mandibular symphysis volume as a source of bone grafting. Clin Implant Dent Relat Res 2010 June 1; 12(2): 99-104

27. Montazem A, Valauri DV, St-Hilaire H, Buchbinder D. The mandibular symphysis as a donor site in maxillofacial bone grafting: a quantitative anatomic study. J Oral Maxillofac Surg 2000; 58(12): 1368-71

28. Verdugo F, Simonian K, Smith McDonald R, Nowzari H. Quantitation of mandibular ramus volume as a source of bone grafting. Clin Implant Dent Relat Res 2009; 11(Suppl 1): 32-37

29. Nkenke E, Radespiel-Tröger M, Wiltfang J, Schultze- Mosgau S, Winkler G, Neukam FW. Morbidity of harvesting of retromolar bone grafts: a prospective study. Clin Oral Impl Res, 13, 2002; 514-521

30. Smith, B.R., Rajchel, J.L., Waite, D.E. \& Read, L. Mandibular anatomy as it relates to rigid fixation of the sagittal ramus split osteotomy. J of Oral Maxillofac Surg, 1991; 49: 222-226 
Figure 1 Representative images from the Galileos CBCT scanner

Figure $\mathbf{2 a}$ and $\mathbf{2 b} \quad$ Radiographic analysis of symphyseal anatomy

A-C Canine tip from outer buccal cortex of mandible

A-B Canine tip to superior aspect incisive canal

H-D Buccal aspect of incisive canal from buccal outer cortex

G-F Maximum thickness of mandible below canine tip

L-M Maximum width of the mandible below the two central incisors

Figure 3a, 3b and 3c Radiographic analysis of retro-molar anatomy

E-F Thickness of mandible at position of IDC

$\mathrm{G}-\mathrm{I}$ Crestal thickness of mandible at second molar position

B-C Depth of IDC from crest of ridge at a position $10 \mathrm{~mm} \pm 0.5 \mathrm{~mm}$ distal to the distal aspect of the lower second molar

D-E Distance between buccal aspect of IDC and outer aspect of buccal plate of mandible

G-H Distance from outer aspect of buccal plate to the root of the mandibular second molar 
Table 1 Radiographic measurements obtained from symphyseal analysis.

\begin{tabular}{|c|c|c|c|c|c|c|}
\hline Measurement & Min. & Max. & Mean & SD & $\begin{array}{c}\text { Females: } \\
\text { Mean }\end{array}$ & $\begin{array}{c}\text { Males: } \\
\text { Mean }\end{array}$ \\
\hline $\begin{array}{c}\text { Maximum width of } \\
\text { mandible at midline (LM) }\end{array}$ & $11.3 \mathrm{~mm}$ & $16.4 \mathrm{~mm}$ & $13.8 \mathrm{~mm}$ & $1.44 \mathrm{~mm}$ & $13.7 \mathrm{~mm}$ & $14.0 \mathrm{~mm}$ \\
\hline $\begin{array}{c}\text { Canine tip from buccal plate } \\
\text { (AC) }\end{array}$ & $1.9 \mathrm{~mm}$ & $7.3 \mathrm{~mm}$ & $4.0 \mathrm{~mm}$ & $1.11 \mathrm{~mm}$ & $4.0 \mathrm{~mm}$ & $3.9 \mathrm{~mm}$ \\
\hline $\begin{array}{c}\text { Canine tip to incisive canal } \\
\text { (AB) }\end{array}$ & $1.2 \mathrm{~mm}$ & $8.9 \mathrm{~mm}$ & $4.3 \mathrm{~mm}$ & $1.59 \mathrm{~mm}$ & $4.1 \mathrm{~mm}$ & $4.5 \mathrm{~mm}$ \\
\hline $\begin{array}{c}\text { Incisive canal from buccal } \\
\text { plate (HD) }\end{array}$ & $1.0 \mathrm{~mm}$ & $6.9 \mathrm{~mm}$ & $3.0 \mathrm{~mm}$ & $1.27 \mathrm{~mm}$ & $2.9 \mathrm{~mm}$ & $3.0 \mathrm{~mm}$ \\
\hline $\begin{array}{c}\text { Max width of mandible } \\
\text { below canine tip (GF) }\end{array}$ & $7.7 \mathrm{~mm}$ & $14.9 \mathrm{~mm}$ & $11.5 \mathrm{~mm}$ & $1.85 \mathrm{~mm}$ & $11.5 \mathrm{~mm}$ & $11.5 \mathrm{~mm}$ \\
\hline
\end{tabular}

Table 2 Radiographic measurements obtained from retromolar analysis.

\begin{tabular}{|c|c|c|c|c|c|c|}
\hline Measurement & Min. & Max. & Mean & SD & $\begin{array}{c}\text { Females: } \\
\text { Mean }\end{array}$ & $\begin{array}{c}\text { Males: } \\
\text { Mean }\end{array}$ \\
\hline $\begin{array}{c}\text { Thickness of mandible } \\
\text { at IDC (EF) }\end{array}$ & $4.7 \mathrm{~mm}$ & $11.1 \mathrm{~mm}$ & $7.6 \mathrm{~mm}$ & $1.47 \mathrm{~mm}$ & $7.7 \mathrm{~mm}$ & $7.4 \mathrm{~mm}$ \\
\hline $\begin{array}{c}\text { Thickness of mandible } \\
\text { at crest (GI) }\end{array}$ & $10.2 \mathrm{~mm}$ & $18.2 \mathrm{~mm}$ & $13.8 \mathrm{~mm}$ & $1.98 \mathrm{~mm}$ & $13.6 \mathrm{~mm}$ & $14.2 \mathrm{~mm}$ \\
\hline $\begin{array}{c}\text { Depth of IDC (BC) } \\
\text { Distance of IDC from } \\
\text { buccal plate (DE) }\end{array}$ & $1.0 \mathrm{~mm}$ & $15.4 \mathrm{~mm}$ & $10.1 \mathrm{~mm}$ & $2.48 \mathrm{~mm}$ & $9.9 \mathrm{~mm}$ & $10.5 \mathrm{~mm}$ \\
\hline $\begin{array}{c}\text { Distance from buccal } \\
\text { plate to second molar } \\
\text { (GH) }\end{array}$ & $1.0 \mathrm{~mm}$ & $6.5 \mathrm{~mm}$ & $3.3 \mathrm{~mm}$ & $1.27 \mathrm{~mm}$ & $3.2 \mathrm{~mm}$ & $3.4 \mathrm{~mm}$ \\
\hline
\end{tabular}

